\title{
Mitochondrial Perturbation Contributing to Cognitive Decline in Streptozotocin- Induced Type 1 Diabetic Rats
}

\author{
Yu Zhou ${ }^{b}$ Siheng Lian ${ }^{a} \quad$ Jin Zhang ${ }^{c}$ Donghai Linc Caihua Huang ${ }^{\mathrm{d}}$ Libin Liu ${ }^{\mathrm{b}}$ \\ Zhou Chen $^{\text {a }}$ \\ aThe School of Pharmacy, Fujian Medical University, Fuzhou, bepartment of Endocrinology, Fujian \\ Medical University Union Hospital, Fuzhou 'Key Laboratory for Chemical Biology of Fujian Province, \\ College of Chemistry and Chemical Engineering, Xiamen University, Xiamen, 'Exercise and Health \\ Laboratory, Xiamen University of Technology, Xiamen, China
}

\section{Key Words}

Type 1 diabetes - Diabetic encephalopathy - Mitochondrial perturbation - Mitochondrial dynamics $•$ Metabolomic analysis

\begin{abstract}
Background/Aims: Mitochondrial perturbation is a well-established cause of cognitive decline, but as yet it is unclear how mitochondria-associated neuronal abnormalities in type 1 diabetic (T1DM) brain contribute to cognitive decline. Methods: The streptozotocin (STZ)-induced mouse model of T1DM was used. The Morris water maze test was applied to assess the effect of T1DM on learning and memory. We detected changes in mitochondrial morphology, function and dynamics. Furthermore, we employed metabolomic analysis to reveal the underlying mechanisms of mitochondrial perturbation which contribute to cognitive decline. Results: Our results show that T1DM impairs mitochondrial dynamics, morphology and function in neurons, associated with a decline in cognitive ability. Metabolomic analyses revealed that T1DM mainly affects metabolic pathways involved in mitochondrial energy failure and impairs the antioxidative system. Conclusion: These results lay the basis for understanding the underlying mitochondria-associated causes of T1DM-associated cognitive decline and may provide a potential treatment strategy for this condition in future.

\section{Introduction}

The prevalence of diabetes mellitus (DM) worldwide has been projected to be 366 million by 2030 according to the World Health Organization (WHO) [1]. As two major subtypes of DM, type 1 diabetes (T1DM) and type 2 diabetes (T2DM) account for approximately 7-12\% and $87-91 \%$ of all diabetic cases respectively [2]. The main feature of T1DM is hyperglycemia and hypoinsulinemia, T2DM is hyperglycemia and insulin resistance. In recent years, there have been major reports suggesting that DM can affect cognitive function [3-5], and that 
patients with DM are at a high risk of developing dementia [6] and Alzheimer's disease (AD) [7, 8]. Adverse changes to the central nervous system (CNS) in diabetes which underlie these pathologies are known as diabetic encephalopathy, which currently has no effective treatment. Compared to other well-studied diabetic complications including retinopathy, nephropathy and cardiovascular diseases, diabetic encephalopathy remains relatively unexplored.

Mitochondria are essential for cell survival, as the main organelle responsible for adenosine triphosphate (ATP) synthesis, calcium homeostasis, and reactive oxygen species (ROS) production and detoxification [9]. In neurons, mitochondria are distributed throughout the axons, presynaptic terminals, and dendrites. Mitochondria play active roles in regulating synaptogenesis and morphological/functional responses to synaptic activity. Diabetes has been reported to elicit AD-like brain changes linked with cognitive decline and neurodegeneration, such as elevated tau expression and phosphorylation and accumulation of amyloid- $\beta(A \beta)$ [10-12]. Both $A \beta$ and tau trigger mitochondrial alterations. This mitochondrial dysfunction can lead to an acute neuronal energy deficit, which in the chronic phase results in modifications to neuronal synapses and neurodegeneration in the diabetic brain [13].

Mitochondria are dynamic organelles that change their morphology through fission and fusion in order to maintain their function. Fission events are regulated by dynamin-related protein (Drp1), and fusion events are regulated by large dynamin-related GTPases known as mitofusin (Mfn) -1 and -2, and optic atrophy 1 (Opa1) [14]. Synaptic mitochondria are vital for maintenance of synaptic function and transmission through normal mitochondrial dynamics. Disruption of mitochondrial dynamics, synaptic damage, and the resultant impairment in cognitive function have proven to be pathological features of T2DM - affected brains $[15,16]$. The importance of mitochondria in supporting synaptic function has made them a promising target for new therapeutic strategies treating diabetic encephalopathy. However, whilst cognitive dysfunction in T1DM and T2DM share many similarities, important differences do exist [17]. So far, evidence regarding the perturbation of mitochondrial morphology, function and dynamics in neurons focuses largely on T2DM, with mitochondrial abnormalities in T1DM little studied. In addition, the mechanisms underlying mitochondrial damage and dysfunction and how these might be addressed are not well understood. Therefore, the main aim of this study was to use a streptozotocin (STZ)-induced animal model of diabetes to evaluate the effects of T1DM on mitochondria in hippocampal neurons. Furthermore, we sought to identify the main metabolic signatures of mitochondrial perturbation using metabolomic analysis to reveal potential mechanisms underlying cognitive decline in T1DM.

\section{Materials and Methods}

\section{Experimental Animals and STZ-Induced Type 1 Diabetic Model}

Thirty male Sprague-Dawley rats (220-250 g) were purchased from the Department of Research Animal Center, Shanghai, China. All animals were housed at Fujian Medical University under controlled temperature, humidity and a 12/12 h dark-light cycle (lights on at 6:00 and off at 18:00) with food and water ad libitum. All experiments were approved by the Fujian Animal Research Ethics Committee.

The T1DM model was induced in rats (DM group; $\mathrm{n}=15$ ) by a single intraperitoneal injection of streptozotocin (STZ; S0130; Sigma, St. Louis, MO, USA) in a 1\% (w/v) solution of $0.1 \mathrm{M}$ citrate buffer (pH 4.24.5 ) at a dose of $50 \mathrm{mg} / \mathrm{kg}$, while 15 age-matched normal control (NC group) rats received injection of the citrate buffer alone. The random blood glucose (RBG) levels of rats were measured daily using a glucometer (Contour Plus, Bayer, Germany) at 3 and 7 days after STZ injection. Rats with RBG $>16.7 \mathrm{mmol} / \mathrm{L}$ for three consecutive tests alongside behavioural markers of diabetes (increased consumption of food and drink, increased urination, decreased weight) were defined as diabetic.

Morris water maze (MWM) test

According to our preliminary work and other similar studies $[18,19]$, rats suffer from cognitive dysfunction at weeks 8-12 after STZ injection. Therefore, the MWM test was performed at 12 weeks after 
STZ injection to assess spatial learning and memory. The MWM test used a circular pool (diameter: $180 \mathrm{~cm}$; height: $50 \mathrm{~cm}$ ) filled with water at $24 \pm 1^{\circ} \mathrm{C}$. A transparent escape platform (diameter: $8 \mathrm{~cm}$ ) was submerged $1 \mathrm{~cm}$ beneath the water and placed at a fixed position at the center of one of the quadrants. The task was performed as described previously [20]. Briefly, in the acquisition phase (days 1-5), all rats underwent four trials per day with a $10 \mathrm{~min}$ intertrial interval, for 5 consecutive training days. Four starting positions, equally spaced along the circumference of the pool, were randomized across the four trials each day. If a rat did not reach the platform within $60 \mathrm{~s}$, it was gently guided there and was allowed to remain on it for $15 \mathrm{~s}$ before being returned to its home cage. The time taken to search for the transparent platform was recorded as the escape latency. On day 6, a probe trial was performed to assess spatial memory retention. During this trial, rats were allowed to swim freely for $60 \mathrm{~s}$, but no platform was present. The time spent in the target quadrant where the escape platform had been located during the acquisition trial was calculated and the number of times that the rat crossed over the target quadrant was also recorded.

\section{Transmission electron microscope (TEM) examination}

The ultrastructure of cells in the hippocampal CA1 region was observed using a TEM (Philips, Amsterdam, Netherlands). Animals were anesthetized with $10 \%(\mathrm{v} / \mathrm{v})$ choral hydrate $(0.4 \mathrm{ml} / 10 \mathrm{~g}$, intraperitoneally). After removing the skull, the brains were rapidly removed and placed on ice. The CA1 region of the hippocampus was dissected. Specimens were fixed by immersion in $3 \%(\mathrm{v} / \mathrm{v})$ buffered glutaraldehyde, then in $1 \%(\mathrm{v} / \mathrm{v})$ osmium tetroxide. Samples were dehydrated in ascending grades of ethanol, embedded in Epon 812, and five random sections were examined under the TEM. Photos were taken of every section, yielding at least 100-200 mitochondria per rat for the morphometry assay analysis. The images (final viewing magnification was approximately 25, 000×) were analyzed using Image Pro Plus 6.0. The TEM operators were blind to the experimental groups of the samples. Mitochondria were analyzed only in the synapses and not in the somata. Synapses were identified by the presence of three or more synaptic vesicles at the presynaptic terminal, parallel pre- and post-synaptic membranes with a discernable synaptic cleft, and a postsynaptic density. The criteria for identifying mitochondria were the presence of distinctive cristae and a double membrane. Then the parameters of mitochondria were quantified by a stereological method [21-24], including the volume density ( $\mathrm{Vv}$ ), the numeric density (Nv), and the specific surface area $(\delta)$.

$$
\begin{aligned}
& V_{v=} \sum_{i=1}^{n} A X_{i} / \sum_{i=1}^{n} A r_{i} \\
& N_{v}=\frac{1}{1.57} \sqrt{\left(\sum_{i=1}^{n} N X_{i} / \sum_{i=1}^{n} A r_{i}\right)^{3} / V_{v}}
\end{aligned}
$$

$$
\delta=4 \sum_{i=1}^{n} B X_{i} / \pi \sum_{i=1}^{n} A r_{i}
$$

where $i$ represents the number of photos; $A x$ represents the area of the mitochondrial cross-sections; $A r$ represents the area of the reference strain; $N x$ represents the number of mitochondrial cross-sections; and $B x$ represents the perimeter of the mitochondrial cross-sections.

\section{Measurement of mitochondrial function}

Mitochondrial fractions of hippocampal tissue were prepared using the Mitochondria Isolation Kit (Beyotime Biotechnology, Jiangsu, China). Briefly, hippocampal tissues were immediately removed and washed by phosphate buffered saline (PBS). Then the tissues were homogenized with the isolation buffer on ice, and centrifugated at $1000 \mathrm{~g}$ for $5 \mathrm{~min}$ at $4{ }^{\circ} \mathrm{C}$. The supernatant was centrifuged again at $3500 \mathrm{~g}$ for 10 $\min$ at $4{ }^{\circ} \mathrm{C}$ to obtain the mitochondrial fractions (deposition). Activities of respiratory chain complex I were measured by NADH-ubiquinone oxidoreductase methods [25, 26]. Mitochondria samples $(0.5 \mathrm{mg} / \mathrm{ml}, 20$ $\mu \mathrm{l})$ were added to $165 \mu \mathrm{l}$ of reaction buffer $(50 \mathrm{mM} \mathrm{KCl}, 10 \mathrm{mM}$ Tris-HCI, $1 \mathrm{mM}$ ethylenediaminetetraacetic acid [EDTA], $2 \mathrm{mM} \mathrm{KCN}, 5 \mu \mathrm{M} \mathrm{CoQ1)}$ and incubated for $5 \mathrm{~min}$ at $30^{\circ} \mathrm{C} .15 \mu \mathrm{l}$ of NADH was added at $75 \mu \mathrm{M}$ 
with acceptors at adequate concentration for maximal activity. The time course of NADH oxidation was followed for $3 \mathrm{~min}$ in a double-wavelength spectrophotometer equipped with a rapid mixing apparatus at an excitation wavelength of $340 \mathrm{~nm}$. The rate of ATP production was determined using an Enhanced ATP Assay Kit (Beyotime Biotechnology) according to the manufacturer's instructions. The concentration of ATP was calculated according to an ATP standard curve and normalized by protein concentration. Values are expressed as fold-change relative to Control.

\section{Quantitative real-time PCR}

Total RNA was extracted from hippocampal tissue using RNAprep Pure Tissue Kit with DNase treatment (Tiangren Biotech, Beijing, China). $1 \mu$ g of total RNA was reverse transcribed using PrimeScript ${ }^{\mathrm{TM}} \mathrm{RT}^{\mathrm{T}}$ reagent Kit (TaKaRa, Beijing, China) according to the manufacturer's instructions. The primers (DNA sequence 5'3') were as follows: for Opa1, forward ACCTTGCCAGTTTAGCTCCC and reverse TTGGGACCTGCAGTGAAGAA; for Mfn 2, forward TGCACCGCCATATAGAGGAAG and reverse TCTGCAGTGAACTGGCAATG; For Drp1, forward ATGCCAGCAAGTCCACAGAA and reverse TGTTCTCGGGCAGACAGTTT; For GAPDH, forward TTCCCGTTCAGCTCTGGG and reverse CCCTGCATCCACTGGTGC. All samples were normalized to GAPDH (forward TTCCCGTTCAGCTCTGGG and reverse CCCTGCATCCACTGGTGC). Quantitative real-time PCR amplifications were carried out in a reaction volume of $25 \mu \mathrm{l}$ on a real-time PCR instrument (Lightcycler 96 , Roche). The 25- $\mu$ l PCR mix was composed of $12.5 \mu \mathrm{l}$ of SYBR PremixEx Taq II (TaKaRa, Beijing, China), $1.0 \mu \mathrm{l}$ of each amplification primer (10 pmol/ $\mu \mathrm{l}), 8.5 \mu \mathrm{l}$ of nucleic acid-free water (TaKaRa, Beijing, China) and $2 \mu \mathrm{l}$ of extract. The thermal cycling comprised an initial denaturing and polymerase hot-start activating step of $3 \mathrm{~min}$ at $95{ }^{\circ} \mathrm{C}$, followed by 40 repeated cycles of $95{ }^{\circ} \mathrm{C}$ for $5 \mathrm{~s}$ and $60{ }^{\circ} \mathrm{C}$ for $30 \mathrm{~s}$. Melting curves were constructed by increasing the final temperature from 60 to $95^{\circ} \mathrm{C}$. All the samples were analyzed in triplicate, and each assay was repeated three times. Each T1DM sample is compared with the 6 controls. Data were analyzed utilizing the comparative cycle threshold $(\mathrm{Ct})$ method $(\Delta \Delta \mathrm{Ct})$. Minimum Information for Publication of Quantitative Real-Time PCR Experiments (MIQE) guidelines were followed [27].

\section{Flow cytometry of isolated mitochondria}

Flow cytometry of isolated mitochondria was measured as previously described [28, 29]. Briefly, mitochondria $(25 \mu \mathrm{g}$ ) of hippocampal tissue were re-suspended in M Buffer (220 mM sucrose, $68 \mathrm{mM}$ mannitol, $10 \mathrm{mM} \mathrm{KCl}, 5 \mathrm{mM} \mathrm{KH}_{2} \mathrm{PO}_{4}, 2 \mathrm{mM} \mathrm{MgCl}_{2}, 500 \mu \mathrm{M}$ ethylene glycol tetraacetic acid, $5 \mathrm{mM}$ succinate, $2 \mu \mathrm{M}$ rotenone, $10 \mathrm{mM}$ 4-(2-hydroxyethyl)-1-piperazineethanesulfonic acid, $\mathrm{pH}$ 7.2, 0.1\% fatty acid-free bovine serum albumin [BSA]). For labelling with antibodies, mitochondria were first blocked with $\mathrm{M}$ buffer supplemented with $10 \%$ fatty acid-free BSA for $15 \mathrm{~min}$ at $4^{\circ} \mathrm{C}$. Mitochondria were then incubated with primary antibody (Drp1, cat. ab56788, 1:100; Abcam; phosphorylation of Drp1 at Serine position 616 (p-Drp1, cat. 3455s, 1:100; Cell Signaling) for $30 \mathrm{~min}$ at $4^{\circ} \mathrm{C}$, washed once with $\mathrm{M}$ buffer and then incubated with PE-anti-rabbit (Cat.8885, 1:500; Cell Signaling) or PE-anti-mouse (Cat.8887, 1:500; Cell Signaling) for $30 \mathrm{~min}$ at $4^{\circ} \mathrm{C}$. Mito-Tracker Green (MTG, $40 \mathrm{nM}$; Beyotime Biotechnology) was used to confirm mitochondrial identity. Ten thousand events were acquired on a FACSVerse flow cytometry system (BD Biosciences). Mitochondria were gated according to light scattering after doublets were excluded, then MTG staining and labelling with Drp1/p-Drp1 were assessed. All flow cytometry data were analyzed with FlowJo 7.6 (Treestar, Ashland, OR, USA). The dyes and antibodies used exhibited distinct spectral properties with minimal to no overlap. Where necessary, compensation was applied according to single-color control samples. An isotype control sample (1:50; Thermo Scientific, Rockford, IL) was also included in every experiment to ensure the specificity of the Drp1/p-Drp1. The percentages of Drp1 and p-Drp1 localized on mitochondria were calculated. All experiments were performed in duplicate, with 3 rats per group used for flow cytometry experiments.

\section{Metabolomic analysis}

Rats were sacrificed by decapitation, and specimens of the hippocampus and cortex were dissected immediately, snap-frozen in liquid nitrogen and stored at $-80^{\circ} \mathrm{C}$ until later use. The frozen tissues $(\mathrm{n}=8$ per group) were weighed and ground by an electric homogenizer in pre-cooled methanol ( $4.000 \mathrm{ml} / \mathrm{g}$ ) for 5 $\min$. Then the homogenate was mixed with chloroform $(4.000 \mathrm{ml} / \mathrm{g})$ and water $(2.854 \mathrm{ml} / \mathrm{g})$ and ground for $2 \mathrm{~min}$. After vortexing for $5 \mathrm{~min}$, the mixture was centrifuged at $12,000 \mathrm{rpm}$ for $15 \mathrm{~min}$ at $4^{\circ} \mathrm{C}$. The aqueous phase was separated from the organic phase and lyophilized for $24 \mathrm{hr}$. Lyophilized intracellular metabolite extracts were resolved in $550 \mu \mathrm{l}$ of sodium phosphate buffer ( $50 \mathrm{mM} \mathrm{K} \mathrm{HPO}_{4} / \mathrm{NaH}_{2} \mathrm{PO}_{4}, \mathrm{pH} 7.4$ ) including 
$10 \% \mathrm{D}_{2} \mathrm{O}, 0.5 \mathrm{mM}$ sodium 3-(trimethylsilyl)propionate-2, 2,3, 3-d4 (TSP) and $0.02 \% \mathrm{NaN}_{3}$, using $\mathrm{D}_{2} \mathrm{O}$ for the field frequency lock, and TSP as the chemical shift reference. All the samples were vortexed adequately and transferred into $5 \mathrm{~mm}$ NMR tubes for further measurement. Then 1D ${ }^{1} \mathrm{H}$ NOESY spectra were acquired on a Bruker Avance III $600 \mathrm{MHz}$ spectrometer at $25^{\circ} \mathrm{C}$ using the pulse sequence [(RD)- $90^{\circ}-\mathrm{t} 1-90^{\circ}-\tau \mathrm{m}-90^{\circ}$ ACQ] (Bruker Biospin pulse program library). Water suppression was achieved by irradiation of the water resonance during the recycle delay of $4 \mathrm{~s}$ and the mixing time $(\mathrm{\tau m})$ of $100 \mathrm{~ms}$. A total of 32 transients with an acquisition time per scan of $2.66 \mathrm{~s}$ were collected in 64,000 data points for each spectrum using a spectral width of $20 \mathrm{ppm}$. Chemical shifts were referenced to the methyl group of TSP at $0 \mathrm{ppm}$. Both phase and baseline corrections were carefully performed.

Data from NMR spectra were preprocessed using the MestReNova 9.0 software (Mestrelab Research, Spain) and MATLAB (Version 2011b; MathWorks, USA). The region of $\delta$ between 5.22-4.67 was excluded to eliminate artifacts related to the residual water resonance in each spectrum. The remaining regions of $\delta$ between 9.40-0.70 were binned in $0.001 \mathrm{ppm}$ intervals and normalized to the integrated area of TSP. Normalized integral values were divided by the masses of corresponding tissue samples to compensate for differences in sample concentration. Data were scaled by Pareto scaling for further multivariate statistical analysis. Principal component analysis (PCA) was performed using SIMCA-P+12.0 (Umetrics, Sweden) to reveal trends in the data and show clusters between samples. The parameters $\mathrm{R}^{2} \mathrm{X}$ (cum) and $\mathrm{Q}^{2}$ (cum) were used to evaluate the quality of the PCA model, where $\mathrm{R}^{2} \mathrm{X}$ (cum) denotes the fraction of the sum of the squares of the explanation of the variables in the model, while Q2 (cum) represents the cross-validated explained variation. Partial least-squares discriminant analysis (PLS-DA) and orthogonal partial least-squares discriminant analysis (OPLS-DA) were subsequently conducted to check grouping trends. To measure the robustness of the PLS-DA model, cross-validation was performed using a random permutation test (200 cycles). The model was considered credible if all the Q2-values on the left were lower than the original point at the right, and the regression line of the Q2-points intersected the vertical axis below zero [30]. We used two criteria derived from the OPLS-DA loading plots to identify differential metabolites significantly responsible for the class separation: the variable importance value (VIP) of the projection [31], and the correlation coefficients ( $r$ ) of the variables. The loading plots were replotted in MATLAB using these two criteria. Variables were color-coded as follows: those with VIP $>1$ and $|r| \geq t$ the critical value of $P=0.01$ were marked in red; those with VIP $>1$ and $|r|$ between the critical values of $\mathrm{P}=0.05$ and $\mathrm{P}=0.01$ were marked in orange; blue indicated variables with VIP $<1$ or $|r|<$ the critical value of $\mathrm{P}=0.05$. Relative concentrations of metabolites were calculated as the integral areas of their corresponding characteristic peaks.

\section{Statistical analysis}

Data from the MWM test were analyzed using a repeated-measure analysis of variance for comparisons between trials, whilst Student's test was used for comparisons between pairs of groups. Statistical analysis was performed using SPSS 18.0 (Chicago, IL, USA), with $\mathrm{p}<0.05$ considered statistically significant.

\section{Results}

Impaired cognitive function in T1DM rats

The MWM test showed that escape latency was not significantly different between the NC and DM rats from day 1 to day 3 during a 5-day training period. However, the escape latencies of the DM rats on days 4 and 5 were significantly longer than those of the NC rats (day 4: $20.3 \pm 5.39$ VS 5.6 \pm 3.61 , p < 0.05; day 5: $18.7 \pm 5.23$ VS $6.2 \pm 2.98, \mathrm{p}<0.05$; Fig. $1 \mathrm{~A}$ ). Fig. 1B shows the swimming trajectory of NC and DM rats in the probe trial of the MWM test. In addition, DM rats made significantly fewer crossings of the target quadrant during the probe trial when compared to $\mathrm{NC}$ rats $(\mathrm{p}<0.001$; Fig. $1 \mathrm{C})$. Therefore, these results demonstrate learning and memory deficits in T1DM rats at $12 \mathrm{wk}$ of age.

Impaired hippocampal mitochondrial morphology, distribution and function in T1DM rats

Given that mitochondria are important for maintenance of synaptic plasticity [16], we examined the changes in hippocampal mitochondrial morphology, distribution and function. As shown in the electron micrographs in Fig. 2A, the mitochondria found in CA1 neurons of $\mathrm{NC}$ rats displayed an intact structure with clear cristae. However in DM rats, although mitochondrial membranes were relatively intact, many cristae within the mitochondria 
were absent. In order to quantify the ultrastructural alterations to synaptic mitochondria, we analyzed a number of morphological parameters, namely the volume density (Vv), numeric density (Nv), and specific surface area $(\delta)$. No difference was found between the $\mathrm{Vv}$ of $\mathrm{NC}$ and DM rats $(p>0.05$; Fig. 2B), whereas $\mathrm{Nv}$ and $\delta$ of mitochondria were lower in DM than $\mathrm{NC}$ rats $(\mathrm{Nv}$ : $\mathrm{p}<$ 0.05 ; Fig. 2 C; $\delta$ : $p<0.01$; Fig. 2D). These results suggest that mitochondria in DM rats were significantly swollen and lower in number. Consistent with these results, DM rats showed a clear reduction in hippocampal ATP level ( $p<0.05$; Fig. 2E) and respiratory chain complex I activity ( $<<0.05$; Fig. 2F) compared to $\mathrm{NC}$ rats. These results suggest that the cognitive decline observed in T1DM rats was associated with impaired hippocampal mitochondrial morphology, distribution and function.

Impaired mitochondrial dynamics in T1DM rats

It has been suggested that mitochondrial dynam-

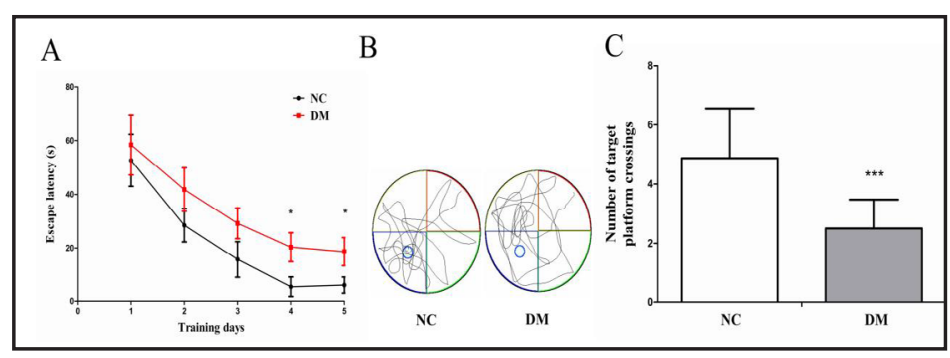

Fig. 1. Learning and memory impairments in DM rats compared to NC rats. (A) Escape latency was significantly longer for DM than NC rats at days 4 and 5; (B) Representative traces of swimming paths from each group in the probe test; (C) DM rats made significantly fewer crossings of the target quadrant during the probe trial when compared to NC rats; $\mathrm{n}=15$ per group; ${ }^{*} \mathrm{p}<0.05 ;{ }^{* * *} \mathrm{p}<0.001$.

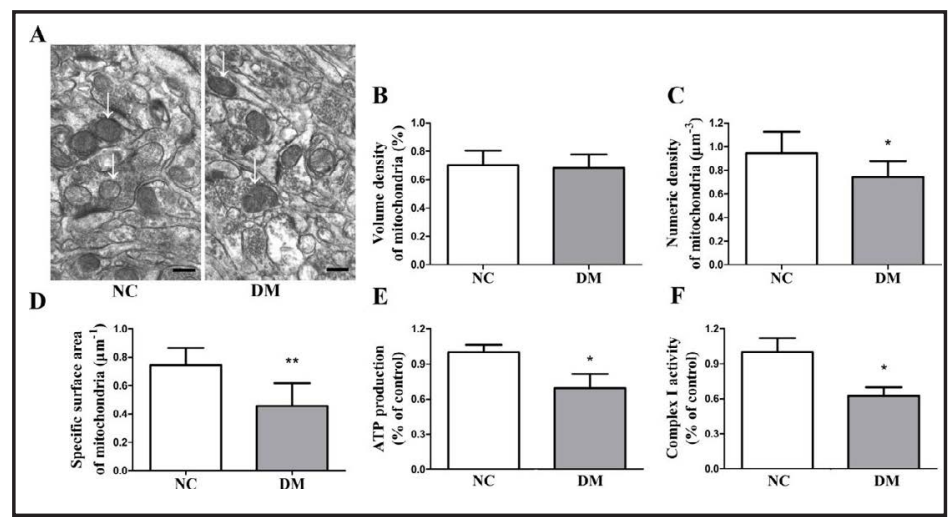

Fig. 2. Impairments in hippocampal mitochondrial morphology, distribution and function in T1DM rats. (A) Morphology of mitochondria in the hippocampus CA1 region. In NC rats, intact mitochondria with clear cristae were found, whereas far fewer cristae were observed in mitochondria from T1DM rats. The white arrowheads indicate mitochondria. Scale bar $=300 \mathrm{~nm}$. No difference was found in the volume densities of mitochondria (B) between the NC and DM groups. Numeric density (C) and specific surface area of mitochondria (D) were lower in DM rats than in NC rats. Mitochondrial ATP production (E) and complex I activity (F) were reduced in the DM compared to NC group; $\mathrm{n}=6$ per group; ${ }^{*} \mathrm{p}<0.05 ;{ }^{* *} \mathrm{p}<0.01$. ics play a critical role in controlling mitochondrial distribution, morphology, and function [16, 32]. To explore the mechanisms underpinning the cognitive impairments in working and long-term memory shown in the MWM, expression levels of genes involved to mitochondrial dynamics were measured in hippocampal homogenates. Quantitative real-time PCR revealed that expression of Opa1 and Mfn2 did not differ significantly between groups, but that Drp1 mRNA levels were increased in DM rats compared to NC rats (Opa1 and Mfn2, p > 0.05; Drp1, p < 0.05; Fig. 3A). However, as a major regulator of mitochondrial fission, Drp1 typically resides in an inactive form in the cytosol and on activation translocates to the mitochondria. In addition, experimental evidence suggests that phosphorylation of Drp1 at S616 is associated with mitochondrial elongation or fragmentation [33].

Therefore, we next determined the levels of Drp1 protein specifically in mitochondria, using a method reported in recent studies that evaluates Drp1 protein levels in the mitochondrial outer membrane by flow cytometry $[28,33]$. In this assay, isolated mitochondria derived from NC and DM rats were first selected/gated according to light scattering proper- 
Fig. 3. Alterations in mitochondrial dynamics in hippocampus of STZinduced T1DM rats. (A) Quantitative real-time PCR revealed that the gene expression of Opa1 and Mfn2 did not differ significantly between DM and NC rats, whereas Drp1 mRNA levels were higher in DM rats compared to $\mathrm{NC}$ rats; $\mathrm{n}=6$ per group; ${ }^{*} \mathrm{P}<0.05$. (B) Isolated mitochondria were first gated by size (forward light scatter, FSC) and granularity (side scatter, SSC). (C) Mitochondria were then selected by staining with MTG (green) a mitochondrial-specific dye, compared with unstained control (grey). (D) Recruitment of activated Drp1 and pDrp1(S616) to mitochondria increases in hippocampi of DM compared to NC rats; $\mathrm{n}=3$ per group; experiments were repeated three times.

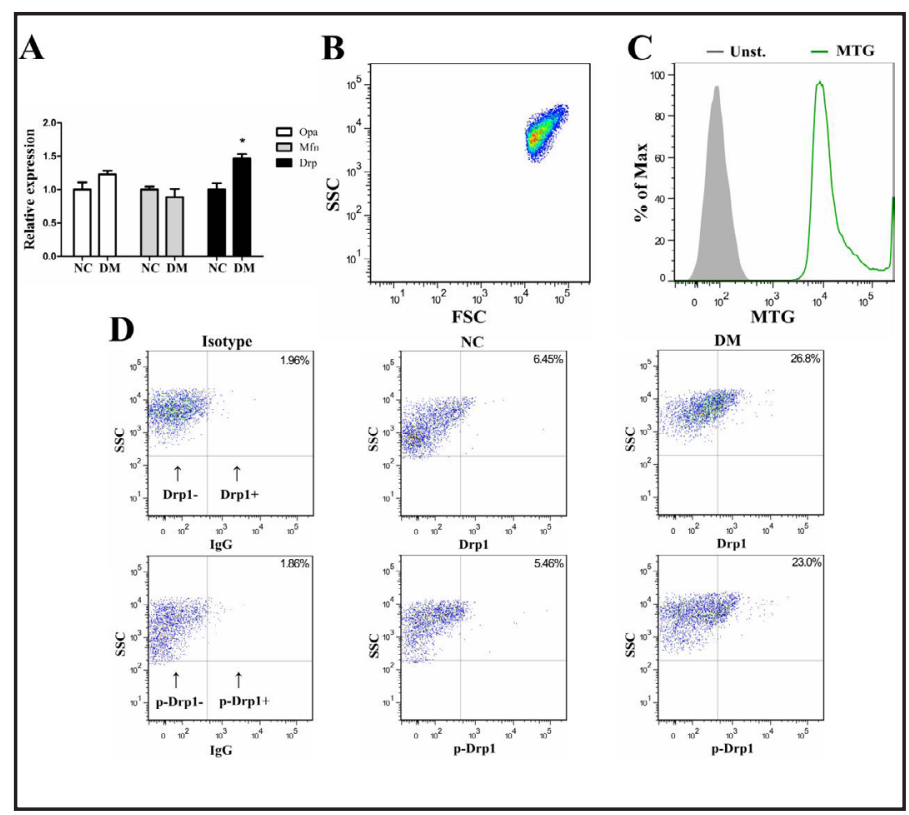

ties (Fig. 3B). We then confirmed mitochondrial identity via positive labelling with the mitochondria-specific, membrane potential independent fluorescent probe MTG (Fig. 3C). These criteria demonstrated that more than $90 \%$ of collected events in each experiment represented mitochondria. We observed increased Drp1 expression in isolated hippocampal mitochondria in DM rats (26.8\%) compared to NC rat $(6.5 \%)$, which was consistent with enhanced translocation of Drp1 to mitochondria following phosphorylation at S616 (23.0\% vs. 5.5\%; Fig. 3D). These data suggest that an imbalance of mitochondrial fission and fusion affects mitochondrial morphology, distribution and function in hippocampus of T1DM rats.
Table 1. Identified metabolites in $1 \mathrm{D}{ }^{1} \mathrm{H}$ NMR spectra of aqueous extracts from hippocampus and cerebral cortex tissues of NC and DM rats. IMP, inosine monophosphate; AMP, adenosine monophosphate; NAD, nicotinamide adenine dinucleotide. Multiplicity: s, singlet; d, double; t, triplet; q, quartet; m, multiple; dd, double of double

\begin{tabular}{|c|c|}
\hline Metabolite & $\delta 1 \mathrm{H}$ and Multiplicity \\
\hline Isoleucine & $0.94(\mathrm{t}), 1.01(\mathrm{~d}), 1.21(\mathrm{~m}), 1.42(\mathrm{~m}), 2.00(\mathrm{~m}), 3.67(\mathrm{~d})$ \\
\hline 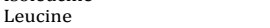 & $0.96(\mathrm{~d}), 0.97(\mathrm{~d}), 1.69(\mathrm{~m}), 1.70(\mathrm{~m}), 1.73(\mathrm{~m}), 3.73(\mathrm{~m})$ \\
\hline Valine & $0.99(\mathrm{~d}), 1.05(\mathrm{~d}), 2.26(\mathrm{~m}), 3.60(\mathrm{~d})$ \\
\hline 3-Hydroxybutyrate & $1.20(\mathrm{~d}), 2.32(\mathrm{dd}), 2.42(\mathrm{dd}), 4.15(\mathrm{~m})$ \\
\hline Threonine & $1.30(\mathrm{~d}), 3.58(\mathrm{~d}), 4.24(\mathrm{~m})$ \\
\hline Lactate & $1.33(\mathrm{~d}), 4.11(\mathrm{q})$ \\
\hline Alanine & $1.47(d), 3.78(q)$ \\
\hline 4-Aminobutyrate & $1.90(\mathrm{~m}), 2.29(\mathrm{t}), 3.01(\mathrm{t})$ \\
\hline Acetate & $1.91(\mathrm{~s})$ \\
\hline $\mathrm{N}$-Acetylaspartate & $2.02(\mathrm{~s}), 2.51(\mathrm{dd}), 2.70(\mathrm{dd}), 4.40(\mathrm{~m}), 7.93(\mathrm{~d})$ \\
\hline Glutamate & $2.08(\mathrm{~m}), 2.12(\mathrm{~m}), 2.34(\mathrm{~m}), 2.37(\mathrm{~m}), 3.75(\mathrm{~m})$ \\
\hline Glutamine & $2.13(\mathrm{~m}), 2.45(\mathrm{~m}), 3.77(\mathrm{t})$ \\
\hline Glutathione & $2.15(\mathrm{~m}), 2.54(\mathrm{~m}), 2.97(\mathrm{dd}), 3.78(\mathrm{~m}), 4.20(\mathrm{q})$ \\
\hline Succinate & $2.40(\mathrm{~s})$ \\
\hline Aspartate & $2.68(\mathrm{dd}), 2.81(\mathrm{dd}), 3.90(\mathrm{dd})$ \\
\hline Creatine & $3.04(\mathrm{~s}), 3.93(\mathrm{~s})$ \\
\hline Phenylalanine & $3.12(\mathrm{dd}), 3.30(\mathrm{dd}), 3.99(\mathrm{dd}), 7.33(\mathrm{~d}), 7.37(\mathrm{t}), 7.43(\mathrm{t})$ \\
\hline Ethanolamine & $3.13(\mathrm{~d}), 3.81(\mathrm{~d})$ \\
\hline Choline & $3.21(\mathrm{~s}), 3.51(\mathrm{dd}), 4.04(\mathrm{t})$ \\
\hline O-Phosphocholine & $3.22(\mathrm{~s}), 3.60(\mathrm{t}), 4.18(\mathrm{~m})$ \\
\hline Glucose & $3.23(\mathrm{~m}), 3.40(\mathrm{~m}), 3.47(\mathrm{~m}), 3.53(\mathrm{dd}), 3.83(\mathrm{~m}), 3.89(\mathrm{dd})$, \\
\hline sn-Glycero-3-phosphocholine & $3.23(\mathrm{~s}), 3.60(\mathrm{dd}), 3.68(\mathrm{dd}), 3.87(\mathrm{~m}), 3.94(\mathrm{~m}), 4.33(\mathrm{~m})$ \\
\hline O-Phosphoethanolamine & $3.24(\mathrm{t}), 4.01(\mathrm{td})$ \\
\hline Taurine & $3.27(\mathrm{t}), 3.43(\mathrm{t})$ \\
\hline myo-Inositol & $3.27(\mathrm{t}), 3.52(\mathrm{dd}), 3.61(\mathrm{t}), 4.05(\mathrm{t})$ \\
\hline Glycerol & $3.55(\mathrm{~m}), 3.64(\mathrm{~m}), 3.78(\mathrm{tt})$ \\
\hline Glycine & $3.57(\mathrm{~s})$ \\
\hline Ascorbate & $3.73(\mathrm{dd})$, \\
\hline Uridine & $3.80(\mathrm{dd}), 3.91(\mathrm{dd}), 4.12(\mathrm{~m}), 4.22(\mathrm{dd}), 4.34(\mathrm{dd}), 5.88(\mathrm{~d}), 5.90(\mathrm{~d}), 7.86(\mathrm{~d})$ \\
\hline Inosine & $3.83(\mathrm{dd}), 3.90(\mathrm{dd}), 4.28(\mathrm{dd}), 4.44(\mathrm{t}), 6.09(\mathrm{~d}), 8.22(\mathrm{~s}), 8.34(\mathrm{~s})$ \\
\hline Serine & $3.84(\mathrm{~m}), 3.94(\mathrm{dd}), 3.98(\mathrm{dd})$ \\
\hline Adenosine & $3.86(\mathrm{dd}), 3.94(\mathrm{dd}), 4.30(\mathrm{q}), 4.44(\mathrm{dd}), 4.81(\mathrm{~s}), 6.02(\mathrm{~d}), 8.12(\mathrm{~s}), 8.28(\mathrm{~s})$ \\
\hline Nicotinurate & $4.00(\mathrm{~s}), 7.60(\mathrm{~m}), 8.25(\mathrm{~m}), 8.71(\mathrm{dd}), 9.00(\mathrm{~m})$ \\
\hline IMP & $4.01(\mathrm{~m}), 4.36(\mathrm{~m}), 4.51(\mathrm{dd}), 4.79(\mathrm{t}), 6.13(\mathrm{~d}), 8.22(\mathrm{~s}), 8.57(\mathrm{~s})$ \\
\hline AMP & $4.01(\mathrm{~m}), 4.36(\mathrm{~m}), 4.51(\mathrm{dd}), 4.79(\mathrm{t}), 6.13(\mathrm{~d}), 8.26(\mathrm{~s}), 8.60(\mathrm{~s})$ \\
\hline NAD & $6.03(\mathrm{~d}), 6.08(\mathrm{~s}), 8.16(\mathrm{~s}), 8.20(\mathrm{~m}), 8.41(\mathrm{~s}), 8.82(\mathrm{~d}), 9.13(\mathrm{~d}), 9.32(\mathrm{~s})$ \\
\hline Fumarate & $6.51(\mathrm{~s})$ \\
\hline Tyrosine & $6.88(d), 7.18(d)$ \\
\hline
\end{tabular}

Effects of T1DM on metabolomic profiles in hippocampus and cerebral cortex

Our results indicate that perturbation of mitochondrial morphology, function and dynamics contributes to cognitive decline in STZ-induced T1DM rats. To determine how T1DM 


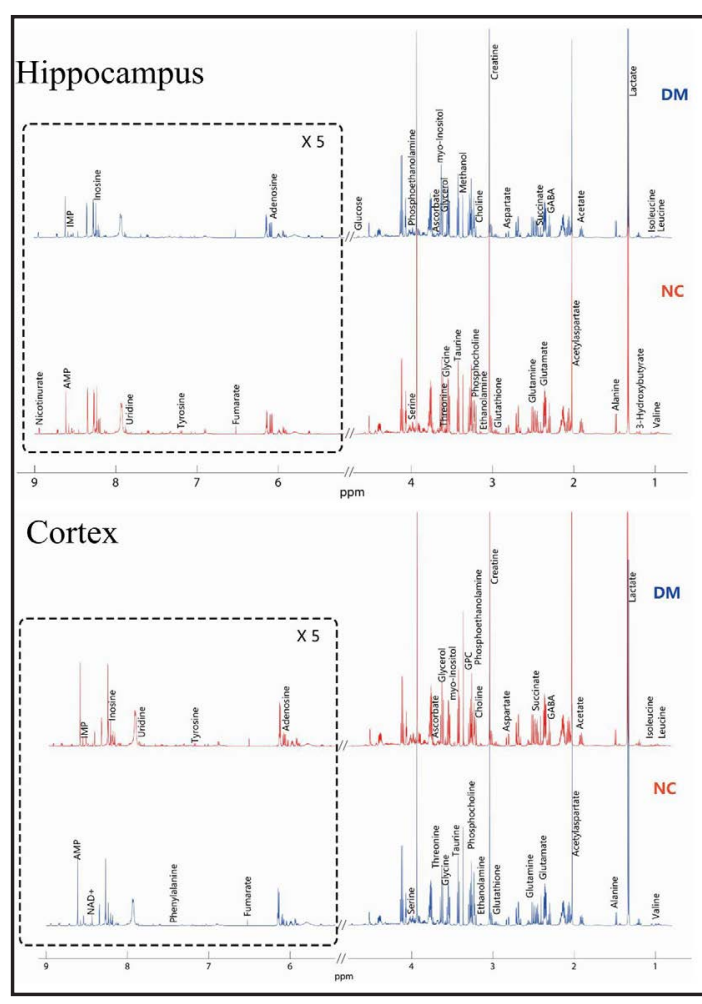

Fig. 4. Representative $1 \mathrm{D}{ }^{1} \mathrm{H}$ NMR spectra of aqueous metabolites derived from hippocampus and cerebral cortex tissues of the $\mathrm{NC}$ and $\mathrm{DM}$ groups.

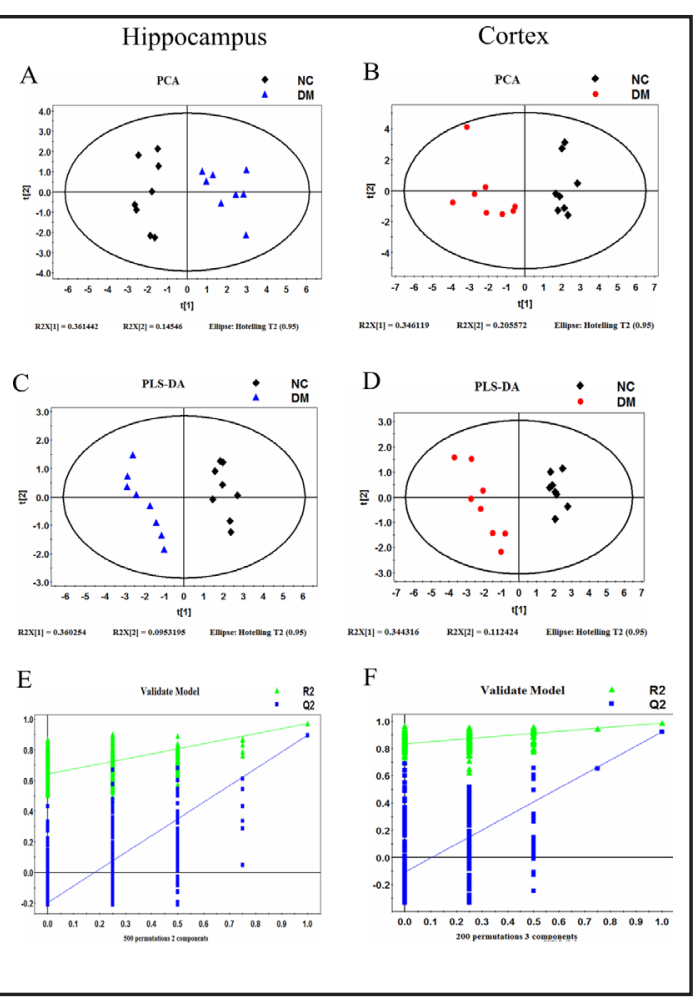

Fig. 5. PCA and PLS-DA analyses based on $1 \mathrm{D}{ }^{1} \mathrm{H}$ NMR spectra of aqueous metabolites derived from the hippocampus and cerebral cortex tissues of the NC and DM groups. (A), (B), PCA score plots of hippocampus (A) and cerebral cortex samples (B); (C), (D), PLS-DA score plots of hippocampus (C) and cerebral cortex samples (D); (E), (F), Permutation tests $(\mathrm{n}=200)$ of PLS-DA models for hippocampus (E) and cerebral cortex samples (F).

affects metabolic pathways involved in mitochondrial perturbation, we analyzed metabolomic profiles in the hippocampus and cerebral cortex of $\mathrm{NC}$ and DM rats. Representative $1 \mathrm{D}^{1} \mathrm{H}$ NMR spectra of the hippocampus and cerebral cortex extracts obtained from NC and DM rats are shown in Fig. 4 and identified metabolites are listed in Table 1. Resonance assignments were confirmed using the Human Metabolome Database (HMDB) (http://www.hmdb.ca/) and the Biological Magnetic Resonance Data Bank (BMRB) (http://www.bmrb.wisc.edu/ metabolomics/). We identified more than 30 metabolites in the hippocampus and cerebral cortex samples, which provided adequate information for assessing variations in metabolic profiles. In order to explore differences in the metabolic profiles, NMR spectra were further segmented and subjected to pattern recognition analysis. An exploratory PCA was applied to obtain a comprehensive comparison between the metabolic profiles of the samples (Fig. $5 \mathrm{~A}$ and $\mathrm{B}$ ). In both the hippocampus and cerebral cortex samples, the score plot of the first two principal components (PC1 and PC2) revealed a clear separation between the DM and NC groups. This suggests that the metabolic characteristics of the two groups were distinctly different in both hippocampus and cerebral cortex. We further analyzed the hippocampus and cerebral cortex data from the two groups using PLS-DA models. Pronounced separations were observed between the DM and NC groups (Fig. 5C and D), indicating their distinctly different metabolic profiles. The PLS-DA models were validated by random permutation tests (Fig. 5E and F). 
Fig. 6. OPLS-DA loading plot based on $1 \mathrm{D}^{1} \mathrm{H}$ NMR spectra. (A), Hippocampus samples. (B), Cerebral cortex samples. The color of the loading plot can be used to identify the level of significance of variables in the class separation: red indicates very significant $(p<0.01)$, orange indicates significant $(\mathrm{p}<0.05)$, and blue indicates non-significant $(\mathrm{p}>$ 0.05).

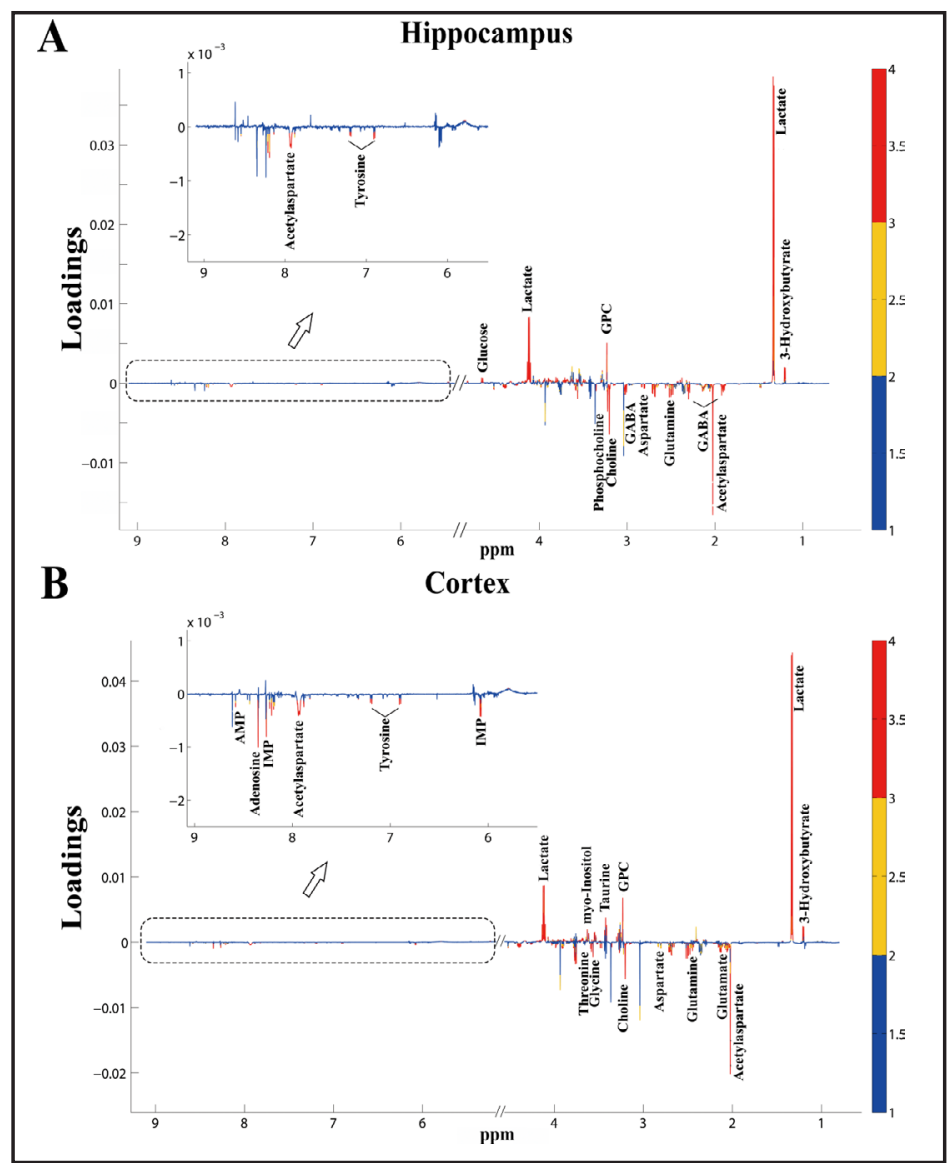

Fig. 7. Comparison of individualized metabolomic profiles and affected metabolic pathways between the NC and DM groups. (A), (B), VIP rank-score of quantified metabolites in the hippocampus and cerebral cortex samples; PC, phosphorylcholine; GPC, glycerolphosphocholine. (C), (D), Metabolic pathways specifically affected in the hippocampus and cerebral cortex samples.

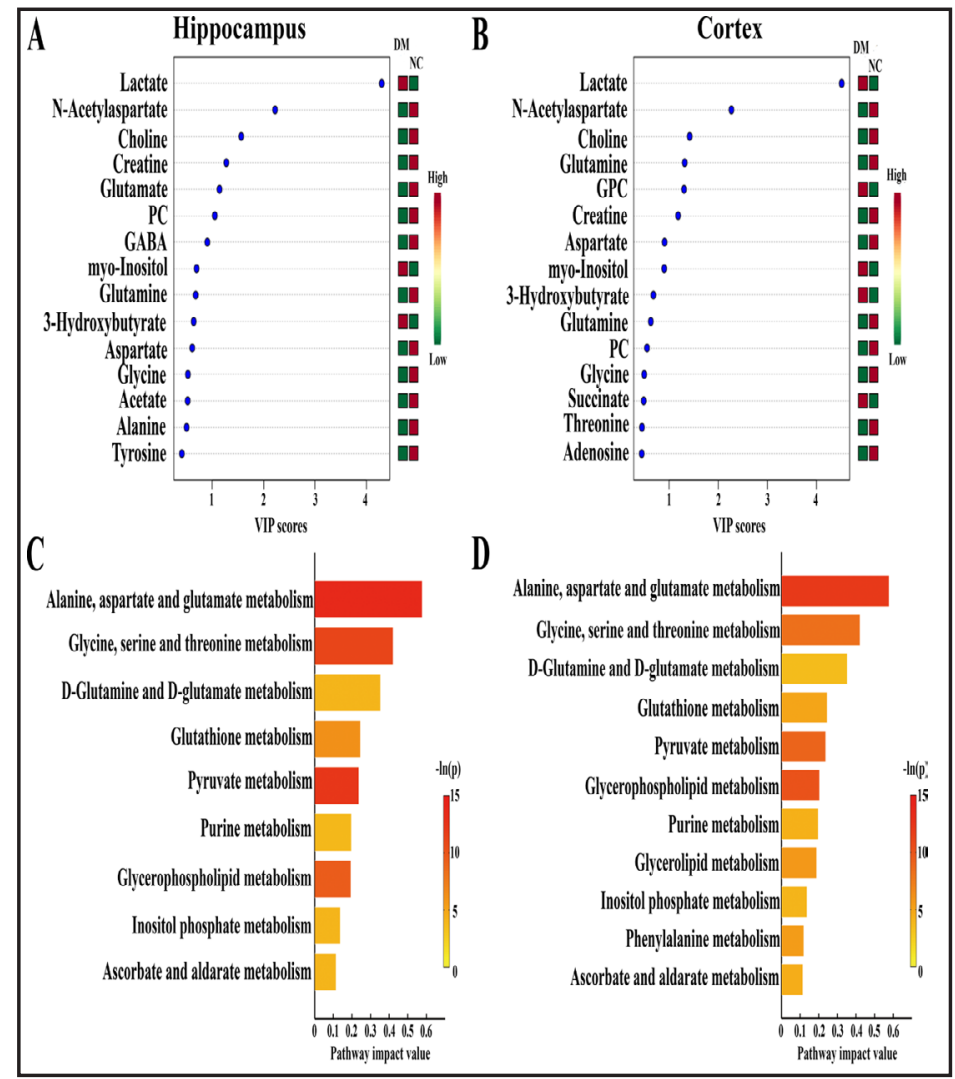




\section{Cellular Physiology Cell Physiol Biochem 2018;46:1668-1682

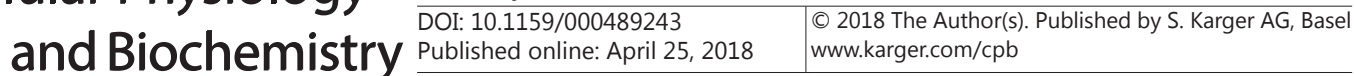 \\ Zhou et al.: Diabetes Induces Mitochondrial Perturbation}

Table 2 summarizes statistically significant changes in relative integral levels of metabolites from the hippocampus and cerebral cortex tissues of NC and DM rats. Fig. $6 \mathrm{~A}$ and $\mathrm{B}$ show the corresponding loading plot with color-coded correlation coefficients (|r|) of OPLS-DA, indicating characteristic metabolites that significantly contributed to the separation between the metabolic profiles of the two groups. The importance of these metabolites in distinguishing metabolic profiles was ranked according to their VIP scores in the OPLS-DA model (Fig.7A and B), showing a huge overlap in identified metabolites between hippocampus and cerebral cortex tissue. Pair-wise comparisons of metabolite levels revealed the presence of characteristic signatures of mitochondrial toxicity, with altered tissue levels of energy metabolites, including lactate, creatine, 3-hydroxybutyrate, succinate, aspartate, alanine, acetate, adenosine. Furthermore, increased levels of glucose, lactate, 3-hydroxybutyrate and decreased levels of $\mathrm{N}$-acetylaspartate (NAA), myo-inositol, creatine, acetate and adenosine in the DM rats strongly suggested the presence of mitochondrial stress and energy dysfunction. Decreased levels of ascorbate and glutathione also revealed that an impairment of mitochondrial antioxidant capacity. Unexpectedly, we found that hippocampus and cerebral cortex tissue had perturbations to very similar metabolic pathways (Fig. 7C and D). These included significant alterations to metabolic pathways involved in energy metabolism, including: alanine, aspartate and glutamate metabolism; pyruvate metabolism; purine metabolism. Antioxidative metabolic pathways, including glutathione metabolism and ascorbate and aldarate metabolism, were also affected. In addition, we identified alterations in neurotransmitter metabolism including: glycine, serine and threonine metabolism; D-Glutamine and D-glutamate metabolism; and phenylalanine metabolism. Changes were also observed in lipid pathways, including metabolism of glycerophospholipid and inositol phosphate. Our metabolic profiling data therefore demonstrate that T1DM mainly affects metabolic pathways involved in mitochondrial energy failure and antioxidative system impairment.

\section{Discussion}

In the current study, we report mitochondrial abnormalities in morphology, function and dynamics in hippocampal neurons that contribute to cognitive decline in the STZinduced T1DM rat model. Application of metabolic profiling permitted the identification of metabolites and metabolic pathways, along with specific metabolic signatures of mitochondrial perturbation associated with T1DM. We believe that the present study may have broader implications for understanding the role of mitochondrial perturbation in synaptic failure and degeneration in T1DM.

Mitochondria are dynamic organelles that change their morphology through fission and fusion in order to maintain their function. It has been proven that increased Drp1/ p-Drp1(S616) levels enhance mitochondrial fragmentation [34] and that excessive 
fragmentation of mitochondria is implicated in the synaptic damage observed in neurodegenerative diseases $[13,35]$ and T2DM $[15,16]$. Although both T1DM and T2DM have been associated with reduced performance in multiple domains of cognitive function and with abnormal brain structure and function in magnetic resonance imaging (MRI) studies, important differences exist. A primary distinguishing feature is that people with T2DM more often do poorly on measures of learning and memory compared to those with T1DM [17]. It is still not known whether or how mitochondrial characteristics change in the neurons of T1DM patients. In this study, we showed that STZ-induced T1DM rats had an impairment in learning and memory at $12 \mathrm{wk}$, as measured on the MWM test. We further demonstrated an increase both in Drp1 mRNA levels and recruitment of activated Drp1 and p-Drp1(S616) protein to mitochondria in hippocampi of T1DM model rats compared to control rats. In agreement with our observations, an earlier study reported increased mitochondrial fission associated with increased protein levels of Drp1 in cortical neurons of STZ-induced T1DM rats [36]. We further confirmed that mitochondrial morphology was altered in hippocampal neurons of T1DM rats using electron microscopy. Measurements of hippocampal mitochondrial function indicated significant reductions in ATP levels and respiratory chain complex I activity. These results suggest that T1DM impairs mitochondrial dynamics and increases mitochondrial fragmentation in hippocampal neurons, which may in turn contribute to cognitive decline.

We used metabolomic analysis to identify perturbed mitochondrial metabolic signatures and reveal the mechanisms underlying mitochondrial dysfunction that may contribute to cognitive decline in T1DM. A major role for mitochondria in energy metabolism is the production of ATP via the tricarboxylic acid (TCA) cycle, electron transfer and oxidative phosphorylation. Although neurons have the receptors necessary to transport extracellular glucose directly, they prefer to use lactate provided by astrocytes. It has been proposed that around $80 \%$ of the total glucose entering the brain from the blood stream does so through astrocytes, and is subsequently converted into lactate for use by all types of brain cells; the other $20 \%$ accounts for the direct uptake of glucose by neurons [37]. In additon, ketone bodies and fatty acids are used as energy substrates under certain circumstances, such as during famine. Glucose and lactate enter mitochondria as pyruvate after undergoing glycolysis in the cytosol. In this study, relative to NC rats, glucose and lactate concentrations were significantly increased in DM rats. In contrast, TCA cycle intermediates such as aspartate and malate did not significantly differ, whereas a ATP levels were significantly lower in T1DM rats relative to controls. These findings suggest that hypometabolism of energy in the T1DM brain is related to impaired use of the substrates of energy metabolism. One study showed that if lactate is not metabolised and eliminated in a timely fashion, high levels of lactate in the brain of $\mathrm{AD}$ can cause cognitive dysfunction [38].

Acetate is considered to be a fatty acid $\beta$-oxidation intermediate; therefore decreased acetate in hippocampus of T1DM rats suggests dysfunction in mitochondrial fatty acid $\beta$-oxidation, a key process that maintains cellular lipid homeostasis and provides energy for cell survival. Disruption of this process leads to bioenergetic failure and intracellular lipid accumulation. This ectopic fatty acid accumulation further inhibits mitochondrial function, leading to increased oxidative stress and apoptotic neuronal cell death in diabetes $[39,40]$. Additionally, elevated levels of ketone bodies (acetoacetate, 3-hydroxybutyrate and acetone) in our study suggest that present of transformation of energy metabolism substrate. Increased ketone body deposition leads to ketosis, and the ketones produced suppress brain glucose consumption [41], further aggravating the energy crisis in the diabetic brain.

Mitochondria play a crucial role in the detoxification of ROS. However, in our study, glutathione and ascorbate levels were decreased significantly in the brains of T1DM rats compared to controls, suggesting that T1DM impairs the antioxidant system in the brain. Mitochondrial ROS production is the main driver of accumulated cellular stress is primarily responsible for diabetes-associated impairments in mitochondrial function, such as dysregulation of calcium homeostasis and ATP synthesis [42]. In turn, defects in energy metabolism at the level of TCA cycle, electron transport chain and oxidative phosphorylation 
further enhance ROS production.

Evidence indicates that NAA is produced in neuronal mitochondria and then used for myelin synthesis in olygodendrocytes [43]. Inositol exists in nine possible stereoisomers, of which myo-inositol is the most widely occurring form in nature. Myo-inositol is a marker of neuroglial cells and exists in the astrocytes of brain tissue [44]. Its phosphorylation status (inositol trisphosphate, $\mathrm{IP}_{3}$ ) controls intracellular calcium concentration, which may play an important role in contributing to various neuronal processes [45]. Increased myo-inositol and decreased NAA were reported to occur in the cortex and hippocampus

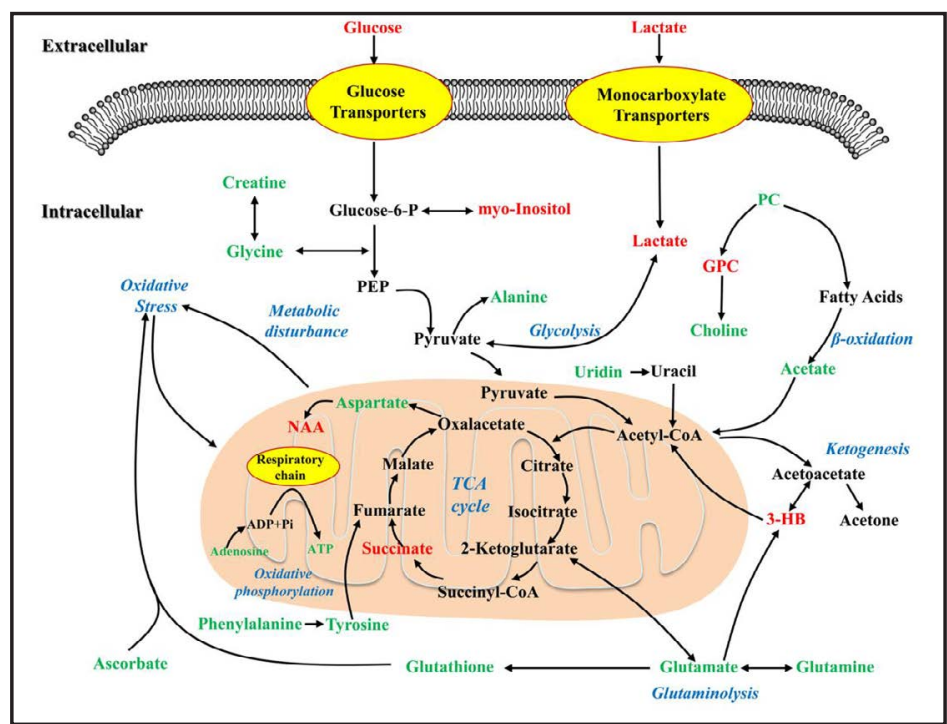

Fig. 8. Schematic of dysregulated metabolic pathways related to pathogenic processes in diabetes-induced mitochondrial dysfunction. Changes in metabolite levels detected by $1 \mathrm{D}{ }^{1} \mathrm{H}$ NMR analysis and metabolic pathways identified by reference to the KEGG database in DM rat brain indicate interactions between metabolic pathways shown are important. Metabolites in red and green represent increased and decreased levels in DM rat brain, respectively, compared with NC rats. 3-HB, 3-Hydroxybutyrate; NAA, N-acetylaspartate; PC, phosphocholine; GPC, glycerolphosphocholin. of $\mathrm{AD}$ mice accompanying amyloid plaques [46-48]. Although changes in these metabolites have been observed in mild cognitive impairment (MCI) compared to healthy controls, they are not yet officially accepted for diagnosis of MCI in clinical settings or for Alzheimer's diagnosis. In our study, decreased levels of NAA and increased levels of myo-inositol strongly suggest that mitochondrial stress occurs in T1DM. In addition, we also identified perturbed neurotransmitter metabolism, including excitatory and inhibitory neurotransmitters. These unexpected results can be interpreted as being a consequence of synaptic dysfunction, mainly due to an insufficient energy supply for neurotransmitter recycling and for maintenance of cellular electrochemical gradients [42]. In summary, the metabolic pathways (referenced to the Kyoto Encyclopedia of Genes and Genomes (KEGG) database) implicated in the pathogenic processes of T1DMinduced mitochondrial dysfunction are shown in Fig. 8.

However, several limitations or further studies should be considered on the basis of the present study. Firstly, we used the mouse primer sequence in the Quantitative real-time PCR section, which is as a possible limitation of the PCR results. Secondly, further investigations are required into whether inhibiting mitochondrial fission can provide neuroprotection for cognitive decline in T1DM. Our results could be built upon by using of Drp1 conditional knockout animals or Drp1 dominant-negative adenoviruses. Thirdly, there is evidence that STZ itself may induce imbalances in brain energy metabolism and other metabolism pathways following intracerebroventricular injection $[49,50]$. Although there is no direct evidence that intraperitoneal injection of STZ affect intracephalic metabolism through the blood brain barrier, the findings of the current study should be confirmed in other animal diabetic models. Lastly, it should also be noted that the results reported here regarding perturbed metabolic pathways do not measure direct interactions between molecules. In future, analysis methods could be used which accurately quantify direct interactions or which measure direct dependencies within regulatory networks. 
In conclusion, our results show that abnormalities in mitochondrial morphology, function and dynamics in hippocampal neurons contribute to cognitive decline in STZinduced T1DM rats. Metabolomic analyses demonstrated that the main effects STZ-induced T1DM were to dysregulate metabolic pathways leading to mitochondrial energy failure and antioxidative system impairment. Our study sheds light on the underlying mechanisms of T1DM-associated cognitive decline from a mitochondrial perspective and could be of benefit in developing future treatments for this disorder.

\section{Acknowledgements}

This work was supported by grants from the Joint Rsearch Program of Fujian Provincial Health and Family Palnning Commision and the Education Department of Fujian Province (WKJ-FJ-12), the Financial Department Special Funds of Fujian Province ([2015]1297) and the Financial Department Special Funds of Fujian Province (2016B020).

\section{Disclosure Statement}

No conflict of interests exists.

\section{References}

1 Wild S, Roglic G, Green A, Sicree R, King H: Global prevalence of diabetes: estimates for the year 2000 and projections for 2030. Diabetes Care 2004;27:1047-1053.

-2 Ogurtsova K, da Rocha Fernandes JD, Huang Y, Linnenkamp U, Guariguata L, Cho NH, Cavan D, Shaw JE, Makaroff LE: IDF Diabetes Atlas: Global estimates for the prevalence of diabetes for 2015 and 2040. Diabetes Res Clin Pract 2017;128:40-50.

3 Crane PK, Walker R, Hubbard RA, Li G, Nathan DM, Zheng H, Haneuse S, Craft S, Montine TJ, Kahn SE, McCormick W, McCurry SM, Bowen JD, Larson EB: Glucose levels and risk of dementia. N Engl J Med 2013;369:540-548.

4 Ding J, Strachan MW, Reynolds RM, Frier BM, Deary IJ, Fowkes FG, Lee AJ, McKnight J, Halpin P, Swa K, Price JF: Diabetic retinopathy and cognitive decline in older people with type 2 diabetes: the Edinburgh Type 2 Diabetes Study. Diabetes 2010;59:2883-2889.

5 Spauwen PJ, Kohler S, Verhey FR, Stehouwer CD, van Boxtel MP: Effects of type 2 diabetes on 12-year cognitive change: results from the Maastricht Aging Study. Diabetes Care 2013;36:1554-1561.

-6 Gonzalez-Reyes RE, Aliev G, Avila-Rodrigues M, Barreto GE: Alterations in Glucose Metabolism on Cognition: A Possible Link Between Diabetes and Dementia. Curr Pharm Des 2016;22:812-818.

7 Yang Y, Song W: Molecular links between Alzheimer's disease and diabetes mellitus. Neuroscience 2013;250:140-150.

-8 van Bussel FC, Backes WH, van Veenendaal TM, Hofman PA, van Boxtel MP, Schram MT, Sep SJ, Dagnelie PC, Schaper N, Stehouwer CD, Wildberger JE, Jansen JF: Functional Brain Networks Are Altered in Type 2 Diabetes and Prediabetes: Signs for Compensation of Cognitive Decrements? The Maastricht Study. Diabetes 2016;65:2404-2413.

-9 Johannsen DL, Ravussin E: The role of mitochondria in health and disease. Curr Opin Pharmacol 2009;9:780-786.

10 Zhao WQ Townsend M: Insulin resistance and amyloidogenesis as common molecular foundation for type 2 diabetes and Alzheimer's disease. Biochim Biophys Acta 2009;1792:482-496.

11 Planel E, Tatebayashi Y, Miyasaka T, Liu L, Wang L, Herman M, Yu WH, Luchsinger JA, Wadzinski B, Duff KE, Takashima A: Insulin dysfunction induces in vivo tau hyperphosphorylation through distinct mechanisms. J Neurosci 2007;27:13635-13648.

12 Jolivalt CG, Hurford R, Lee CA, Dumaop W, Rockenstein E, Masliah E: Type 1 diabetes exaggerates features of Alzheimer's disease in APP transgenic mice. Exp Neurol 2010;223:422-431. 


\section{Cellular Physiology Cell Physiol Biochem 2018;46:1668-1682 \begin{tabular}{l|l} 
and Biochemistry DOI: 10.1159/000489243 & $\begin{array}{l}\text { (c) 2018 The Author(s). Published by S. Karger AG, Basel } \\
\text { www.karger.com/cpb }\end{array}$
\end{tabular}

13 Akhter F, Chen D, Yan SF, Yan SS: Mitochondrial Perturbation in Alzheimer's Disease and Diabetes. Prog Mol Biol Transl Sci 2017;146:341-361.

14 Yoon Y, Galloway CA, Jhun BS, Yu T: Mitochondrial dynamics in diabetes. Antioxid Redox Signal 2011;14:439-457.

-15 Edwards JL, Quattrini A, Lentz SI, Figueroa-Romero C, Cerri F, Backus C, Hong Y, Feldman EL: Diabetes regulates mitochondrial biogenesis and fission in mouse neurons. Diabetologia 2010;53:160-169.

-16 Huang S, Wang Y, Gan X, Fang D, Zhong C, Wu L, Hu G, Sosunov AA, McKhann GM, Yu H, Yan SS: Drp1mediated mitochondrial abnormalities link to synaptic injury in diabetes model. Diabetes 2015;64:17281742.

17 McCrimmon RJ, Ryan CM, Frier BM: Diabetes and cognitive dysfunction. Lancet 2012;379:2291-2299.

18 Rostami S, Momeni Z, Behnam-Rassouli M, Rooholamin S: A comparative study on the effects of type I and type II diabetes on learning and memory deficit and hippocampal neuronal loss in rat. Minerva Endocrinol 2013;38:289-295.

19 Ma LY, Fei YL, Wang XY, Wu SD, Du JH, Zhu M, Jin L, Li M, Li HL, Zhai JJ, Ji LP, Ma RR, Liu SF, Li M, Ma L, Ma XR, Qu QM, Lv YL: The Research on the Relationship of RAGE, LRP-1, and Abeta Accumulation in the Hippocampus, Prefrontal Lobe, and Amygdala of STZ-Induced Diabetic Rats. J Mol Neurosci 2017;62:1-10.

20 Vorhees CV, Williams MT: Morris water maze: procedures for assessing spatial and related forms of learning and memory. Nat Protoc 2006;1:848-858.

-21 Xu XW, Shi C, He ZQ, Ma CM, Chen WH, Shen YP, Guo Q, Shen CJ, Xu J: Effects of phytoestrogen on mitochondrial structure and function of hippocampal CA1 region of ovariectomized rats. Cell Mol Neurobiol 2008;28:875-886.

22 Balietti M, Giorgetti B, Di Stefano G, Casoli T, Platano D, Solazzi M, Bertoni-Freddari C, Aicardi G, Lattanzio F, Fattoretti P: A ketogenic diet increases succinic dehydrogenase (SDH) activity and recovers age-related decrease in numeric density of SDH-positive mitochondria in cerebellar Purkinje cells of late-adult rats. Micron 2010;41:143-148.

23 Bertoni-Freddari C, Fattoretti P, Casoli T, Spagna C, Meier-Ruge W, Ulrich J: Morphological plasticity of synaptic mitochondria during aging. Brain Res 1993;628:193-200.

-24 Balietti M, Giorgetti B, Casoli T, Solazzi M, Tamagnini F, Burattini C, Aicardi G, Fattoretti P: Early selective vulnerability of synapses and synaptic mitochondria in the hippocampal CA1 region of the $\operatorname{Tg} 2576$ mouse model of Alzheimer's disease. J Alzheimers Dis 2013;34:887-896.

25 Estornell E, Fato R, Pallotti F, Lenaz G: Assay conditions for the mitochondrial NADH:coenzyme Q oxidoreductase. FEBS letters 1993;332:127-131.

26 Xu LH, Xie H, Shi ZH, Du LD, Wing YK, Li AM, Ke Y, Yung WH: Critical Role of Endoplasmic Reticulum Stress in Chronic Intermittent Hypoxia-Induced Deficits in Synaptic Plasticity and Long-Term Memory. Antioxidants \& redox signaling 2015;23:695-710.

27 Bustin SA, Benes V, Garson JA, Hellemans J, Huggett J, Kubista M, Mueller R, Nolan T, Pfaffl MW, Shipley GL, Vandesompele J, Wittwer CT: The MIQE guidelines: minimum information for publication of quantitative real-time PCR experiments. Clin Chem 2009;55:611-622.

28 Pickles S, Arbour N, Vande Velde C: Immunodetection of outer membrane proteins by flow cytometry of isolated mitochondria. Journal of visualized experiments : JoVE 2014:51887.

-29 Pickles S, Destroismaisons L, Peyrard SL, Cadot S, Rouleau GA, Brown RH, Jr., Julien JP, Arbour N, Vande Velde C: Mitochondrial damage revealed by immunoselection for ALS-linked misfolded SOD1. Human molecular genetics 2013;22:3947-3959.

-30 Eriksson L, Andersson PL, Johansson E, Tysklind M: Megavariate analysis of environmental QSAR data. Part I--a basic framework founded on principal component analysis (PCA), partial least squares (PLS), and statistical molecular design (SMD). Mol Divers 2006;10:169-186.

-31 Nguyen DV, Rocke DM: Tumor classification by partial least squares using microarray gene expression data. Bioinformatics 2002;18:39-50.

-32 Ding M, Dong Q Liu Z, Liu Z, Qu Y, Li X, Huo C, Jia X, Fu F, Wang X: Inhibition of dynamin-related protein 1 protects against myocardial ischemia-reperfusion injury in diabetic mice. Cardiovascular diabetology 2017;16:19.

-33 Zhang L, Trushin S, Christensen TA, Bachmeier BV, Gateno B, Schroeder A, Yao J, Itoh K, Sesaki H, Poon WW, Gylys KH, Patterson ER, Parisi JE, Diaz Brinton R, Salisbury JL, Trushina E: Altered brain energetics induces mitochondrial fission arrest in Alzheimer's Disease. Sci Rep 2016;6:18725. 


\section{Cellular Physiology Cell Physiol Biochem 2018;46:1668-1682 \begin{tabular}{ll|l} 
and Biochemistry Published online: April 25, 2018 & $\begin{array}{l}\text { (c) } 2018 \text { The Author(s). Published by S. Karger AG, Basel } \\
\text { www.karger.com/cpb }\end{array}$ \\
\hline
\end{tabular}

34 Reddy PH, Reddy TP, Manczak M, Calkins MJ, Shirendeb U, Mao P: Dynamin-related protein 1 and mitochondrial fragmentation in neurodegenerative diseases. Brain Res Rev 2011;67:103-118.

-35 Bertholet AM, Delerue T, Millet AM, Moulis MF, David C, Daloyau M, Arnaune-Pelloquin L, Davezac N, Mils V, Miquel MC, Rojo M, Belenguer P: Mitochondrial fusion/fission dynamics in neurodegeneration and neuronal plasticity. Neurobiol Dis 2016;90:3-19.

-36 Santos RX, Correia SC, Alves MG, Oliveira PF, Cardoso S, Carvalho C, Duarte AI, Santos MS, Moreira PI: Insulin therapy modulates mitochondrial dynamics and biogenesis, autophagy and tau protein phosphorylation in the brain of type 1 diabetic rats. Biochim Biophys Acta 2014;1842:1154-1166.

-37 Hyder F, Patel AB, Gjedde A, Rothman DL, Behar KL, Shulman RG: Neuronal-glial glucose oxidation and glutamatergic-GABAergic function. J Cereb Blood Flow Metab 2006;26:865-877.

-38 Liguori C, Stefani A, Sancesario G, Sancesario GM, Marciani MG, Pierantozzi M: CSF lactate levels, tau proteins, cognitive decline: a dynamic relationship in Alzheimer's disease. J Neurol Neurosurg Psychiatry 2015;86:655-659.

39 Schrauwen P, Hesselink MK: Oxidative capacity, lipotoxicity, and mitochondrial damage in type 2 diabetes. Diabetes 2004;53:1412-1417.

40 Choi J, Ravipati A, Nimmagadda V, Schubert M, Castellani RJ, Russell JW: Potential roles of PINK1 for increased PGC-1alpha-mediated mitochondrial fatty acid oxidation and their associations with Alzheimer disease and diabetes. Mitochondrion 2014;18:41-48.

41 LaManna JC, Salem N, Puchowicz M, Erokwu B, Koppaka S, Flask C, Lee Z: Ketones suppress brain glucose consumption. Adv Exp Med Biol 2009;645:301-306.

42 Duarte JM: Metabolic Alterations Associated to Brain Dysfunction in Diabetes. Aging Dis 2015;6:304-321.

-43 Duarte JM, Lei H, Mlynarik V, Gruetter R: The neurochemical profile quantified by in vivo 1H NMR spectroscopy. Neuroimage 2012;61:342-362.

-44 Chen SQ, Wang PJ, Ten GJ, Zhan W, Li MH, Zang FC: Role of myo-inositol by magnetic resonance spectroscopy in early diagnosis of Alzheimer's disease in APP/PS1 transgenic mice. Dement Geriatr Cogn Disord 2009;28:558-566.

45 Berridge MJ: Inositol trisphosphate and calcium signalling mechanisms. Biochim Biophys Acta 2009;1793:933-940.

-46 Oberg J, Spenger C, Wang FH, Andersson A, Westman E, Skoglund P, Sunnemark D, Norinder U, Klason T, Wahlund LO, Lindberg M: Age related changes in brain metabolites observed by 1H MRS in APP/PS1 mice. Neurobiol Aging 2008;29:1423-1433.

47 Marjanska M, Curran GL, Wengenack TM, Henry PG, Bliss RL, Poduslo JF, Jack CR, Jr., Ugurbil K, Garwood M: Monitoring disease progression in transgenic mouse models of Alzheimer's disease with proton magnetic resonance spectroscopy. Proc Natl Acad Sci U S A 2005;102:11906-11910.

48 von Kienlin M, Kunnecke B, Metzger F, Steiner G, Richards JG, Ozmen L, Jacobsen H, Loetscher H: Altered metabolic profile in the frontal cortex of PS2APP transgenic mice, monitored throughout their life span. Neurobiol Dis 2005;18:32-39.

49 Lannert H, Hoyer S: Intracerebroventricular administration of streptozotocin causes long-term diminutions in learning and memory abilities and in cerebral energy metabolism in adult rats. Behav Neurosci 1998;112:1199-1208.

50 Grieb P: Intracerebroventricular Streptozotocin Injections as a Model of Alzheimer's Disease: in Search of a Relevant Mechanism. Mol Neurobiol 2016;53:1741-1752. 